\title{
Discrete point symmetries of ordinary differential equations
}

\author{
B Y P. E. HYDON \\ Department of Mathematics and Statistics, University of Surrey, \\ Guildford, Surrey GU2 5XH, UK
}

Received 2 June 1997; revised 16 September 1997; accepted 20 November 1997

This paper describes a method that enables the user to construct systematically the set of all discrete point symmetries of a given ordinary differential equation (ODE) of order two or greater, provided that the ODE has at least a one-parameter Lie group of point symmetries.

The method is easy to use, and is based upon Lie's method of constructing continuous symmetries. The calculations are simple, and computer algebra is not usually required. Various examples are used to illustrate the method. The paper concludes with a proof that every ODE whose Lie group of point symmetries is isomorphic to the unimodular group has at least four inequivalent real discrete point symmetries.

Keywords: differential equations; symmetry methods; constructive techniques; equivariant bifurcation theory; boundary-value problems; computer algebra

\section{Introduction}

Symmetries of all kinds are valuable in the study of ordinary differential equations (ODEs). Many ODEs of physical importance have (local) Lie groups of point symmetries that can be found systematically (see Olver 1986; Bluman \& Kumei 1989; Stephani 1989). These continuous symmetries can be used to reduce the order of an ODE or to locate separatrices and other topologically important solutions (Bluman \& Kumei 1989).

Discrete point symmetries are commonly used to reduce the domain on which an ODE is solved numerically, thereby increasing computational efficiency. This is possible if the ODE, the computational domain and the boundary conditions are invariant. For example, the following boundary-value problem (BVP) describes the deformation of a loaded elastic beam on an inhomogeneous elastic support; the beam is simply supported at its ends:

$$
\begin{gathered}
y^{(i v)}+k(x) y=p(x), \quad x \in(-1,1), \\
y(-1)=y^{\prime \prime}(-1)=y(1)=y^{\prime \prime}(1)=0 .
\end{gathered}
$$

Here $y(x)$ is the deformation at the point $x$, the loading is described by $p(x)$ and $k(x)$ measures the reaction per unit deformation of the elastic support. If both $k$ and $p$ are even functions of $x$, then the BVP (1.1) has a discrete symmetry: it is invariant under the transformation

$$
\Gamma:(x, y) \mapsto(-x, y) .
$$

If $k(x)$ is such that there exists a unique solution of (1.1), then that solution is invariant under the action of $\Gamma$. It can be computed from the reduced problem

$$
\begin{gathered}
y^{(i v)}+k(x) y=p(x), \quad x \in(0,1), \\
y^{\prime}(0)=y^{\prime \prime \prime}(0)=y(1)=y^{\prime \prime}(1)=0 .
\end{gathered}
$$

Proc. R. Soc. Lond. A (1998) 454, 1961-1972

Printed in Great Britain 
Alternatively, the solution of (1.1) can be computed using a spectral method, with trial functions that are invariant under $\Gamma$. With either approach, using the discrete symmetry greatly improves the efficiency of the computation.

Many nonlinear BVPs have more than one solution. If a given BVP is parametrized, bifurcation theory can be used to analyse the variation in the number and nature of solutions as the parameter(s) vary. Symmetries of the BVP may have a marked effect, and must be taken into account. This is accomplished by using equivariant bifurcation theory (see Golubitsky et al. 1988), which requires that all symmetries (discrete and continuous) are known. Various applications to physical problems are described in Allgower et al. $(1992 a, b)$ and Chadam et al. (1996). Some BVPs have hidden discrete symmetries. These are symmetries of the differential equation that fail to map the original BVP to itself, but which leave an extended problem invariant (see Crawford et al. 1991). The system cannot be analysed correctly unless the hidden discrete symmetries are taken into account. For the remainder of the current paper, attention is focused on differential equations rather than BVPs. The reason for this approach is that the set of point symmetries (hidden or otherwise) of a particular BVP is a subset of the set of all point symmetries of the governing ODE.

Discrete point symmetries are typically much harder to find than continuous point symmetries. Some progress can be made by using a suitable ansatz (Gaeta \& Rodríguez 1996), but this approach does not guarantee that all discrete point symmetries of a given ODE have been found. The main difficulty is that point symmetries of ODEs are determined by a coupled system of nonlinear partial differential equations (PDEs). Lie's method works by linearizing this system, which is why it is relatively easy to calculate all continuous point symmetries. Reid et al. (1993) used a differential analogue of Buchberger's algorithm to obtain a Gröbner basis for the nonlinear determining equations for a particular second-order ODE. This method is computationally intensive and requires computer algebra, but when it works, it yields all discrete point symmetries.

The current paper outlines a new method that determines all discrete point symmetries of a given ODE systematically and simply, without the need for computer algebra. It is applicable to any ODE with an $N$-parameter Lie group of point symmetries $(N \geqslant 1)$, for which the Lie symmetry generators can be found by the standard method. Most ODEs of physical interest fall into this category, as does the example used by Reid et al. (1993) to demonstrate the Gröbner basis method.

As well as being simple to apply to a given ODE, the new method can be used to prove general results about the existence and nature of discrete point symmetries. For instance, we prove the somewhat surprising result that every ODE whose group of Lie point symmetries is isomorphic to $S L(2)$ has at least four discrete point symmetries that cannot be mapped to one another by any of the continuous point symmetries. In other words, some discrete point symmetries are generic.

\section{Point symmetries of ODEs}

We begin by recalling some basic facts about point symmetries of ODEs. A point symmetry of the ODE

$$
y^{(n)}=\omega\left(x, y, y^{\prime}, \ldots, y^{(n-1)}\right), \quad n \geqslant 2,
$$

is a smooth invertible transformation of the $(x, y)$ plane that maps the set of solutions of (2.1) to itself. Specifically, a point symmetry is a diffeomorphism

$$
\Gamma:(x, y) \mapsto(\hat{x}(x, y), \hat{y}(x, y))
$$

Proc. R. Soc. Lond. A (1998) 
that is extended to derivatives by the recursive formula

$$
\Gamma: y^{(k)} \mapsto \hat{y}^{(k)} \equiv \frac{\mathrm{d} \hat{y}^{(k-1)}}{\mathrm{d} \hat{x}}, \quad \text { where } \quad \hat{y}^{(0)}=\hat{y},
$$

such that

$$
\hat{y}^{(n)}=\omega\left(\hat{x}, \hat{y}, \hat{y}^{\prime}, \ldots, \hat{y}^{(n-1)}\right) \quad \text { when }(2.1) \text { holds. }
$$

The composition of any two point symmetries is a point symmetry.

Many ODEs have point symmetries which form (local) Lie groups with oneparameter Lie subgroups. These symmetries can be written in the form

$$
\begin{aligned}
& \hat{x}=x+\epsilon \xi(x, y)+O\left(\epsilon^{2}\right)=\mathrm{e}^{\epsilon X} x, \\
& \hat{y}=y+\epsilon \eta(x, y)+O\left(\epsilon^{2}\right)=\mathrm{e}^{\epsilon X} y,
\end{aligned}
$$

for each $\epsilon$ in some neighbourhood of zero, where $X$ is the infinitesimal generator

$$
X=\xi(x, y) \partial_{x}+\eta(x, y) \partial_{y}
$$

The set of all infinitesimal generators of one-parameter Lie groups of point symmetries of an ODE is a Lie algebra, $\mathcal{L}$. Henceforth, attention is restricted to ODEs $(2.1)$ for which $\mathcal{L}$ is non-trivial. Lie's method is used to construct a basis for the Lie algebra, $\left\{X_{i}\right\}_{i=1}^{N}$; then the structure constants, $c_{i j}^{k}$, are obtained from the commutator relations

$$
\left[X_{i}, X_{j}\right]=X_{i} X_{j}-X_{j} X_{i}=c_{i j}^{k} X_{k} .
$$

(The usual summation convention is used in (2.7) and henceforth.) Now suppose that $\Gamma:(x, y) \mapsto(\hat{x}, \hat{y})$ is a symmetry of $(2.1)$; then

$$
\hat{\Gamma}_{i}(\epsilon)=\Gamma \mathrm{e}^{\epsilon X_{i}} \Gamma^{-1}
$$

is also a symmetry for each $\epsilon$ in some neighbourhood of zero. By definition,

$$
\hat{\Gamma}_{i}(\epsilon)=\mathrm{e}^{\epsilon \Gamma X_{i} \Gamma^{-1}},
$$

and so $\hat{\Gamma}_{i}(\epsilon)$ is a one-parameter local Lie group whose infinitesimal generator is

$$
\hat{X}_{i}=\Gamma X_{i} \Gamma^{-1}
$$

Therefore $\hat{X}_{i} \in \mathcal{L}$ for each $i$. We calculate $\hat{X}_{i}$ explicitly, as follows:

$$
\hat{X}_{i}=\left(\hat{X}_{i} \hat{x}\right) \partial_{\hat{x}}+\left(\hat{X}_{i} \hat{y}\right) \partial_{\hat{y}}=\left(\Gamma X_{i} x\right) \partial_{\hat{x}}+\left(\Gamma X_{i} y\right) \partial_{\hat{y}}=\xi_{i}(\hat{x}, \hat{y}) \partial_{\hat{x}}+\eta_{i}(\hat{x}, \hat{y}) \partial_{\hat{y}} .
$$

Hence the generators $\left\{\hat{X}_{i}\right\}_{i=1}^{N}$ are simply the basis generators $\left\{X_{i}\right\}_{i=1}^{N}$ with $(x, y)$ replaced by $(\hat{x}, \hat{y})$. Thus they are linearly independent and form a basis for $\mathcal{L}$, and so each $X_{i}$ can be written as a linear combination of the $\hat{X}_{i}$. Furthermore, the structure constants are preserved by the transformation $X_{i} \mapsto \hat{X}_{i}$ :

$$
\left[\hat{X}_{i}, \hat{X}_{j}\right]=c_{i j}^{k} \hat{X}_{k} \quad \text { when }(2.7) \text { holds. }
$$

In other words, the mapping is a symmetry of the Lie algebra. These results are summarized in the following lemma.

Lemma 2.1. Every point symmetry $\Gamma$ of (2.1) induces an automorphism of the Lie algebra $\mathcal{L}$ of generators of one-parameter local Lie groups of point symmetries of (2.1). For each such $\Gamma$, there exists a constant non-singular $N \times N$ matrix $\left(b_{i}^{l}\right)$ such that

$$
X_{i}=b_{i}^{l} \hat{X}_{l}
$$

Proc. R. Soc. Lond. A (1998) 
The automorphism preserves all structure constants.

Lemma 2.1 provides a tool for calculating the discrete point symmetries of a given ODE, once the Lie algebra has been calculated. The most direct approach is discussed in the next section; this approach must be used when $\mathcal{L}$ is abelian (i.e. when all structure constants are zero). A neater version of the method should be used when $\mathcal{L}$ is non-abelian; this is introduced in $\S 4$.

\section{The basic method}

The method has two stages. To begin with, apply lemma 2.1 to obtain the following first-order PDEs which every point symmetry (2.2) of the ODE (2.1) must satisfy:

$$
\left.\begin{array}{ll}
X_{i} \hat{x}=b_{i}^{l} \hat{X}_{l} \hat{x}=b_{i}^{l} \xi_{l}(\hat{x}, \hat{y}), & i=1, \ldots, N, \\
X_{i} \hat{y}=b_{i}^{l} \hat{X}_{l} \hat{y}=b_{i}^{l} \eta_{l}(\hat{x}, \hat{y}), & i=1, \ldots, N .
\end{array}\right\}
$$

These can be solved by the method of characteristics to obtain $(\hat{x}, \hat{y})$ in terms of $x, y, b_{i}^{l}$ and some unknown constants or functions obtained on integration of (3.1). Then $\hat{y}^{(k)}$ can be calculated using (2.3). Note that the solutions of (3.1) always include the trivial symmetry $(\hat{x}, \hat{y})=(x, y)$, which corresponds to $b_{i}^{l}=\delta_{i}^{l}$.

Every point symmetry satisfies (3.1), but there may be solutions of (3.1) that are not point symmetries. The second stage is to see which of these solutions correspond to point symmetries of the ODE, by substituting $\hat{x}, \hat{y}, \hat{y}^{\prime}, \ldots, \hat{y}^{(n)}$ into $(2.4)$ and checking to see whether or not (2.1) is satisfied.

This two-stage process gives a complete list of the point symmetries of (2.1). However, we already know about the Lie point symmetries, so the calculations may be simplified by factoring them out whenever possible. Then we obtain a list of inequivalent discrete symmetries that are not related to one another by any point symmetry generated by a one-parameter Lie group.

It is usually convenient to work in a canonical coordinate system, in which one of the generators $\left(X_{1}\right.$, say) is a translation. This is especially useful when $N=$ $\operatorname{dim}(\mathcal{L})=1$. Canonical coordinates $r(x, y), s(x, y)$ satisfy

$$
X_{1} r=0, \quad X_{1} s=1,
$$

so that

$$
X_{1}=\partial_{s} .
$$

If $\operatorname{dim}(\mathcal{L})=1$ then, writing $\hat{r}=r(\hat{x}, \hat{y})$ and $\hat{s}=s(\hat{x}, \hat{y})$, we obtain from lemma 2.1 the pair of PDEs

$$
\hat{r}_{s}=0, \quad \hat{s}_{s}=b_{1}^{1} \neq 0,
$$

whose general solution is

$$
\hat{r}=f(r), \quad \hat{s}=b_{1}^{1} s+g(r)
$$

for some functions $f$ and $g$. We require that $\Gamma$ is a diffeomorphism, and therefore $f$ and $g$ must be smooth, with $\mathrm{d} f / \mathrm{d} r \neq 0$. The condition that this transformation should be a symmetry determines which functions $f, g$ and constants $b_{1}^{1}$ are allowable.

To illustrate the method, consider the ODE

$$
y^{\prime \prime}=\frac{y^{\prime}}{x}+\frac{4 y^{2}}{x^{3}}
$$

Proc. R. Soc. Lond. A (1998) 
used by Reid et al. (1993) to demonstrate the application of a differential analogue of Buchberger's algorithm. This ODE has a one-dimensional Lie algebra of point symmetry generators, with a basis

$$
X_{1}=x \partial_{x}+y \partial_{y}
$$

In terms of canonical coordinates

$$
r=y / x, \quad s=\ln |x|,
$$

the ODE is equivalent to

$$
\frac{\mathrm{d}^{2} r}{\mathrm{~d} s^{2}}=4 r^{2}+r
$$

The solution (3.5) gives

$$
\frac{\mathrm{d}^{2} \hat{r}}{\mathrm{~d} \hat{s}^{2}}=\frac{b_{1}^{1}\left(\dot{f} r^{\prime \prime}+\ddot{f} r^{\prime 2}\right)+(\ddot{f} \dot{g}-\dot{f} \ddot{g}) r^{\prime 3}}{\left(b_{1}^{1}+\dot{g} r^{\prime}\right)^{3}},
$$

where $\cdot=\mathrm{d} / \mathrm{d} r$ and ${ }^{\prime}=\mathrm{d} / \mathrm{d} s$. The symmetry condition is that

$$
\frac{\mathrm{d}^{2} \hat{r}}{\mathrm{~d} \hat{s}^{2}}=4 \hat{r}^{2}+\hat{r} \quad \text { when }(3.9) \text { holds, }
$$

and therefore

$$
\frac{b_{1}^{1}\left(\dot{f}\left(4 r^{2}+r\right)+\ddot{f} r^{\prime 2}\right)+(\ddot{f} \dot{g}-\dot{f} \ddot{g}) r^{\prime 3}}{\left(b_{1}^{1}+\dot{g} r^{\prime}\right)^{3}}=4 f^{2}+f .
$$

Equating powers of $r^{\prime}$ and using the fact that $b_{1}^{1}$ and $\dot{f}$ are both non-zero gives

$$
\dot{g}=0, \quad f=c_{1} r+c_{2} \quad\left(c_{1} \neq 0\right),
$$

where the $c_{i}$ are constants and

$$
\frac{c_{1}}{\left(b_{1}^{1}\right)^{2}}\left(4 r^{2}+r\right)=4\left(c_{1}\right)^{2} r^{2}+\left(8 c_{2}+1\right) c_{1} r+c_{2}\left(4 c_{2}+1\right) .
$$

The action of the one-parameter Lie group is factored out by taking $g=0$. The remaining possibilities are obtained by equating powers of $r$ in (3.14); they give

$$
(\hat{r}, \hat{s}) \in\left\{(r, s),(r,-s),\left(-r-\frac{1}{4}, \mathrm{i} s\right),\left(-r-\frac{1}{4},-\mathrm{i} s\right)\right\} .
$$

Note that these symmetries form a group, generated by $\Gamma_{1}:(r, s) \mapsto\left(-r-\frac{1}{4}\right.$, is $)$, that is isomorphic to $\mathbb{Z}_{4}$. These discrete symmetries, when written in terms of $x, y$ and composed with scalings generated by $X_{1}$ (with complex $\epsilon$ ) yield the four families of symmetries described by Reid et al. (1993). Moreover, the above method finds the complete list of symmetries very easily, without the need for computer algebra to reduce a complicated system of nonlinear PDEs to a manageable form. Note that if we restrict attention to real-valued symmetries and real $\epsilon$, only the first two of (3.15) are admissible. However, scalings that multiply $x$ and $y$ by a positive factor are distinguished from scalings by a negative factor. Hence the group of real discrete point symmetries is

$$
(\hat{x}, \hat{y}) \in\left\{(x, y),(-x,-y),\left(1 / x, y / x^{2}\right),\left(-1 / x,-y / x^{2}\right)\right\},
$$

which is isomorphic to $\mathbb{Z}_{2} \times \mathbb{Z}_{2}$.

As a second example, consider the ODE

$$
y^{\prime \prime}=\tan y^{\prime},
$$

Proc. R. Soc. Lond. A (1998) 
whose only Lie point symmetries are translations. Here $\mathcal{L}$ is two dimensional, with a basis

$$
X_{1}=\partial_{x}, \quad X_{2}=\partial_{y} .
$$

Equations (3.1) amount to

$$
\left[\begin{array}{ll}
\hat{x}_{x} & \hat{y}_{x} \\
\hat{x}_{y} & \hat{y}_{y}
\end{array}\right]=\left[\begin{array}{ll}
b_{1}^{1} & b_{1}^{2} \\
b_{2}^{1} & b_{2}^{2}
\end{array}\right]\left[\begin{array}{ll}
1 & 0 \\
0 & 1
\end{array}\right],
$$

whose general solution is

$$
(\hat{x}, \hat{y})=\left(b_{1}^{1} x+b_{2}^{1} y+c_{1}, b_{1}^{2} x+b_{2}^{2} y+c_{2}\right), \quad c_{1}, c_{2} \text { const. }
$$

Bearing in mind that $\operatorname{det}\left(b_{i}^{l}\right) \neq 0$, the action of the translation group generated by $X_{1}$ and $X_{2}$ can be factored out by taking $c_{1}=c_{2}=0$. The transformations are extended to the first and second derivatives, yielding

$$
\hat{y}^{\prime}=\frac{b_{1}^{2}+b_{2}^{2} y^{\prime}}{b_{1}^{1}+b_{2}^{1} y^{\prime}}, \quad \hat{y}^{\prime \prime}=\frac{y^{\prime \prime}\left(b_{1}^{1} b_{2}^{2}-b_{1}^{2} b_{2}^{1}\right)}{\left(b_{1}^{1}+b_{2}^{1} y^{\prime}\right)^{3}} .
$$

By equating powers of $y^{\prime}$, etc., it is found that the symmetry condition

$$
\hat{y}^{\prime \prime}=\tan \hat{y}^{\prime} \quad \text { when } \quad y^{\prime \prime}=\tan y^{\prime}
$$

is satisfied if and only if

$$
B=\left[\begin{array}{cc}
1 & k \pi \\
0 & \alpha
\end{array}\right], \quad \alpha \in\{-1,1\}, \quad k \in \mathbb{Z},
$$

where $B$ is used henceforth to denote $\left(b_{i}^{l}\right)$. So the discrete symmetries are all of the form

$$
(\hat{x}, \hat{y})=(x, \alpha y+k \pi x), \quad \alpha \in\{-1,1\}, \quad k \in \mathbb{Z} .
$$

The above examples illustrate the basic method, which is easy to use if $\mathcal{L}$ is low dimensional. However, with increasing $N$, the determining equations can be complicated, because there are many unknown constants $b_{i}^{l}$ to consider. For non-abelian Lie algebras, it is possible to simplify the matrix $B$ before solving the determining equations, making the method much easier to apply.

\section{The improved method}

If $\mathcal{L}$ is not abelian, then at least some of the equations (2.7) are non-trivial. From (2.11), the generators $\hat{X}_{i}$ satisfy the same commutator relations as $X_{i}$, i.e.

$$
\left[\hat{X}_{i}, \hat{X}_{j}\right]=c_{i j}^{k} \hat{X}_{k}
$$

Combining (2.7), (2.13) and (4.1) gives the useful result

$$
c_{l m}^{n} b_{i}^{l} b_{j}^{m}=c_{i j}^{k} b_{k}^{n} \text {. }
$$

It is sufficient to restrict attention to equation (4.2) with $i<j$, because the structure constants are antisymmetric in the two lower indices.

Although (4.2) provides some simplification, it is more effective when it is used in combination with the adjoint action of the Lie group generated by each $X_{j}$. The adjoint action

$$
\operatorname{Ad}\left(\exp \left(\epsilon_{j} X_{j}\right)\right) X_{i}=X_{i}-\epsilon_{j}\left[X_{j}, X_{i}\right]+\left(\epsilon_{j}^{2} / 2 !\right)\left[X_{j},\left[X_{j}, X_{i}\right]\right]-\cdots=a_{i}^{p}\left(\epsilon_{j}, j\right) X_{p}
$$

Proc. R. Soc. Lond. A (1998) 
enables us to factor out those symmetries that are equivalent to one another under a transformation $\mathrm{e}^{\epsilon X}$, for some $X \in \mathcal{L}$ (see Olver 1986). Specifically, the system (2.13) is equivalent to

$$
X_{i}=\tilde{b}_{i}^{l} \hat{X}_{l}
$$

under the group generated by $X_{j}$, where

$$
\tilde{b}_{i}^{l}=a_{i}^{p}\left(\epsilon_{j}, j\right) b_{p}^{l} \text {. }
$$

A straightforward calculation shows that equation (4.2) is invariant under the mapping $b_{i}^{l} \mapsto \tilde{b}_{i}^{l}$, and therefore we will drop tildes as soon as the transformation has been made. By using each generator $X_{j}$ in turn, we obtain a set of inequivalent matrices $B=\left(b_{i}^{l}\right)$, without having to solve any PDEs. This is the set of symmetries of the Lie algebra (up to continuous group equivalence). It is usually possible to ensure that most of the entries in these matrices are zero.

For example, consider the two-dimensional non-abelian Lie algebra $\mathfrak{a}(1)$. We choose a basis $\left\{X_{1}, X_{2}\right\}$ such that $\left[X_{1}, X_{2}\right]=X_{1}$. The only non-zero structure constants are

$$
c_{12}^{1}=-c_{21}^{1}=1 \text {. }
$$

Therefore (4.2) gives

and hence

$$
b_{1}^{1} b_{2}^{2}-b_{1}^{2} b_{2}^{1}=b_{1}^{1}, \quad 0=b_{1}^{2},
$$

$$
B=\left[\begin{array}{ll}
b_{1}^{1} & 0 \\
b_{2}^{1} & 1
\end{array}\right], \quad b_{1}^{1} \neq 0 .
$$

This can be simplified further by factoring out the equivalent symmetries using (4.3) and (4.5). Let $A(j)$ denote the matrix whose components are $a_{i}^{p}\left(\epsilon_{j}, j\right)$. Equation (4.3) gives (after a short calculation)

$$
A(1)=\left[\begin{array}{cc}
1 & 0 \\
-\epsilon_{1} & 1
\end{array}\right], \quad A(2)=\left[\begin{array}{cc}
\mathrm{e}^{\epsilon_{2}} & 0 \\
0 & 1
\end{array}\right] .
$$

Applying (4.5), first with $j=1, \epsilon_{1}=b_{2}^{1} / b_{1}^{1}$, then with $j=2, \epsilon_{2}=-\ln \left|b_{1}^{1}\right|$, we obtain

$$
B=\left[\begin{array}{ll}
\alpha & 0 \\
0 & 1
\end{array}\right], \quad \text { where } \alpha \in\{-1,1\} .
$$

The reduced form of the matrix $B$ is specific to this particular Lie algebra, and is independent of the ODE whose Lie point symmetries are generated by the algebra.

It is important to realize that the order in which the matrices $A(j)$ are used does not affect the classification of the matrices $B$; any ordering gives the same final form, provided that the parameters $\epsilon_{j}$ are chosen appropriately.

Having found the reduced matrices, it is easy to determine the discrete symmetries of ODEs with this Lie algebra. For example, the ODE

$$
y^{\prime \prime \prime}=\frac{y^{\prime \prime 2}}{x}-\frac{y^{\prime \prime}}{y^{\prime}}
$$

has a two-dimensional Lie algebra of point symmetry generators, spanned by

$$
X_{1}=\partial_{y}, \quad X_{2}=\frac{1}{2} x \partial_{x}+y \partial_{y} ;
$$

Proc. R. Soc. Lond. A (1998) 
note that $\left[X_{1}, X_{2}\right]=X_{1}$. So the inequivalent discrete symmetries satisfy

$$
\left[\begin{array}{ll}
X_{1} \hat{x} & X_{1} \hat{y} \\
X_{2} \hat{x} & X_{2} \hat{y}
\end{array}\right]=\left[\begin{array}{cc}
\alpha & 0 \\
0 & 1
\end{array}\right]\left[\begin{array}{cc}
0 & 1 \\
\frac{1}{2} \hat{x} & \hat{y}
\end{array}\right]=\left[\begin{array}{cc}
0 & \alpha \\
\frac{1}{2} \hat{x} & \hat{y}
\end{array}\right],
$$

whose general solution is

$$
\hat{x}=c_{1} x, \quad \hat{y}=\alpha y+c_{2} x^{2}, \quad c_{i} \text { const. }
$$

Substituting (4.14) into the ODE, we find that $\alpha c_{1}^{2}=1$ and $c_{2}=0$ are required for the symmetry condition to be satisfied. Therefore the only discrete point symmetries (up to equivalence) are

$$
(\hat{x}, \hat{y}) \in\{(x, y),(\mathrm{i} x,-y),(-x, y),(-\mathrm{i} x,-y)\},
$$

which is isomorphic to $\mathbb{Z}_{4}$, with group generator

$$
\Gamma_{1}:(x, y) \mapsto(\mathrm{i} x,-y) .
$$

As another example of the method, consider the Chazy equation

$$
y^{\prime \prime \prime}=2 y y^{\prime \prime}-3 y^{\prime 2}+\lambda\left(6 y^{\prime}-y^{2}\right)^{2},
$$

whose symmetries have been studied by Clarkson \& Olver (1996). The Chazy equation has a three-dimensional Lie algebra of Lie point symmetry generators, with a basis

$$
X_{1}=\partial_{x}, \quad X_{2}=x \partial_{x}-y \partial_{y}, \quad X_{3}=x^{2} \partial_{x}-(2 x y+6) \partial_{y} .
$$

The commutators of these generators are

$$
\left[X_{1}, X_{2}\right]=X_{1}, \quad\left[X_{1}, X_{3}\right]=2 X_{2}, \quad\left[X_{2}, X_{3}\right]=X_{3},
$$

and therefore $\mathcal{L}$ is isomorphic to $\mathfrak{s l}(2)$, which is not solvable. However, each of the two-dimensional subalgebras $\operatorname{Span}\left(X_{1}, X_{2}\right)$ and $\operatorname{Span}\left(X_{2}, X_{3}\right)$ are solvable, and so each can be used to reduce (4.17) to a first-order ODE. Surprisingly, each of the subalgebras gives rise to the same first-order ODE if the most natural differential invariants are used for the reduction of order. Indeed, Clarkson (1995, personal communication) observed that the one-parameter groups generated by $X_{1}$ and $X_{3}$ can be used to reduce the Chazy equation to the same second-order ODE.

To explain this result, it is necessary to examine the discrete symmetries of (4.17). First, we identify the inequivalent matrices $B$ associated with $\mathfrak{s l}(2)$. The non-zero structure constants are

$$
c_{12}^{1}=-c_{21}^{1}=1, \quad c_{13}^{2}=-c_{31}^{2}=2, \quad c_{23}^{3}=-c_{32}^{3}=1,
$$

and therefore the adjoint action of the Lie point symmetries is given by

$$
A(1)=\left[\begin{array}{ccc}
1 & 0 & 0 \\
-\epsilon_{1} & 1 & 0 \\
\epsilon_{1}^{2} & -2 \epsilon_{1} & 1
\end{array}\right], \quad A(2)=\left[\begin{array}{ccc}
\mathrm{e}^{\epsilon_{2}} & 0 & 0 \\
0 & 1 & 0 \\
0 & 0 & e^{-\epsilon_{2}}
\end{array}\right], \quad A(3)=\left[\begin{array}{ccc}
1 & 2 \epsilon_{3} & \epsilon_{3}^{2} \\
0 & 1 & \epsilon_{3} \\
0 & 0 & 1
\end{array}\right] \text {. }
$$

As $\mathcal{L}$ is not solvable, it is helpful to begin the classification of matrices $B$ by using the adjoint action.

If $b_{1}^{1} \neq 0$, then we can use $A(1)$ to set $b_{2}^{1}=0$, by taking $\epsilon_{1}=b_{2}^{1} / b_{1}^{1}$. Then the Proc. R. Soc. Lond. A (1998) 
identities (4.2) give

$$
B=\left[\begin{array}{ccc}
b_{1}^{1} & b_{1}^{2} & b_{1}^{3} \\
0 & 1 & b_{2}^{3} \\
0 & 0 & 1 / b_{1}^{1}
\end{array}\right] .
$$

Now use $A(3)$ with $\epsilon_{3}=-b_{1}^{1} b_{2}^{3}$ to set $b_{2}^{3}=0$. Then (4.2) gives $b_{1}^{2}=b_{1}^{3}=0$. Finally, use $A(2)$ with $\epsilon_{2}=-\ln \left|b_{1}^{1}\right|$ to obtain two possibilities:

$$
B=\left[\begin{array}{lll}
\alpha & 0 & 0 \\
0 & 1 & 0 \\
0 & 0 & \alpha
\end{array}\right], \quad \alpha \in\{-1,1\} .
$$

The only remaining possibility is that $b_{1}^{1}=0$. Applying a similar procedure to the above, we find that in this case

$$
B=\left[\begin{array}{ccc}
0 & 0 & \alpha \\
0 & -1 & 0 \\
\alpha & 0 & 0
\end{array}\right], \quad \alpha \in\{-1,1\} .
$$

So there are four distinct matrices $B$ associated with $\mathfrak{s l}(2)$; in other words, this Lie algebra has four symmetries (up to equivalence).

For the Chazy equation, the system (3.1) is

$$
\left[\begin{array}{cc}
X_{1} \hat{x} & X_{1} \hat{y} \\
X_{2} \hat{x} & X_{2} \hat{y} \\
X_{3} \hat{x} & X_{3} \hat{y}
\end{array}\right]=B\left[\begin{array}{cc}
1 & 0 \\
\hat{x} & -\hat{y} \\
\hat{x}^{2} & -(2 \hat{x} \hat{y}+6)
\end{array}\right],
$$

where $B$ is one of the four matrices (4.23). It turns out that there are two solutions of (4.24) for each $B$. For example, if $B$ is the identity matrix, then

$$
(\hat{x}, \hat{y}) \in\{(x, y),(x+(6 / y),-y)\} .
$$

However, the second of these is not a symmetry of the Chazy equation, and must therefore be discarded. Each matrix $B$ generates precisely one discrete symmetry of the Chazy equation; the complete list is

$$
(\hat{x}, \hat{y}) \in\left\{(x, y),(-x,-y),\left(-1 / x, x^{2} y+6 x\right),\left(1 / x,-\left(x^{2} y+6 x\right)\right)\right\} .
$$

Each of these symmetries is an involution: it is its own inverse. In particular,

$$
(x, y) \mapsto(\hat{x}, \hat{y})=\left(-1 / x, x^{2} y+6 x\right)
$$

exchanges $X_{1}$ and $X_{3}$; it corresponds to $(4.23 b)$ with $\alpha=1$. The fundamental differential invariants associated with the one-parameter group generated by $X_{1}$ are

$$
r_{1}=y, \quad v_{1}=y^{\prime},
$$

in terms of which the Chazy equation is equivalent to

$$
v_{1}^{\prime \prime}=\frac{2 r_{1} v_{1}^{\prime}-\left(v_{1}^{\prime}\right)^{2}}{v_{1}}-3+\lambda\left(6-\frac{r_{1}^{2}}{v_{1}}\right)^{2} .
$$

The point transformation (4.27) maps $\left(r_{1}, v_{1}\right)$ to $\left(r_{3}, v_{3}\right)$, where

$$
r_{3}=\hat{y}=x^{2} y+6 x, \quad v_{3}=\hat{y}^{\prime}=x^{4} y^{\prime}+2 x^{3} y+6 x^{2} ;
$$

Proc. R. Soc. Lond. A (1998) 
these are fundamental differential invariants associated with $X_{3}$. The above transformation is a symmetry, and therefore

$$
v_{3}^{\prime \prime}=\frac{2 r_{3} v_{3}^{\prime}-\left(v_{3}^{\prime}\right)^{2}}{v_{3}}-3+\lambda\left(6-\frac{r_{3}^{2}}{v_{3}}\right)^{2} .
$$

Equation (4.31) can also be obtained directly from (4.17). This explains Clarkson's observation that reducing the Chazy equation with invariants either of $X_{1}$ or of $X_{3}$ yields the same second-order ODE.

Indeed, the result can be generalized as follows. Suppose that an ODE has two one-parameter Lie groups of point symmetries, generated by $X_{i}, i=1,2$, with corresponding fundamental differential invariants $r_{i}(x, y)$ and $v_{i}\left(x, y, y^{\prime}\right)$. Each pair of differential invariants $\left(r_{1}, v_{1}\right)$ or $\left(r_{2}, v_{2}\right)$ can be used to reduce the order of the original ODE by one. Suppose also that the reduced ODE has the same form whichever of the pairs is used. Then there is a transformation mapping $X_{1}$ to $X_{2}$ such that $\left(r_{1}, v_{1}\right) \mapsto\left(r_{2}, v_{2}\right)$. This transformation is a symmetry of the original ODE, because it is a symmetry of the reduced ODE.

\section{Existence of discrete symmetries}

The set of inequivalent discrete symmetries with $b_{i}^{l}=\delta_{i}^{l}$ is a normal subgroup, $G_{0}$, of the group $G$ of inequivalent discrete symmetries of an ODE. In this section we consider the factor group $F=G / G_{0}$. By construction, each matrix $B$ generates at most one element of $F$. Is it possible to state any general results about inequivalent discrete symmetries of families of ODEs that share the same Lie algebra?

For example, the Chazy equation has one real discrete symmetry corresponding to each of the four matrices (4.23). We now show that every ODE whose Lie algebra of point symmetry generators is isomorphic to $\mathfrak{s l}(2)$ has at least one real discrete symmetry for each of the matrices (4.23). The inequivalent actions of $S L(2)$ on the real plane have been completely classified (see Clarkson \& Olver 1996). Any ODE whose Lie algebra of point symmetry generators is $\mathfrak{s l}(2)$ can be mapped by a real point transformation to an ODE with one of the following sets of generators:

$$
\begin{gathered}
X_{1}=\partial_{x}, \quad X_{2}=x \partial_{x}, \quad X_{3}=x^{2} \partial_{x} \\
X_{1}=\partial_{x}, \quad X_{2}=x \partial_{x}+y \partial_{y}, \quad X_{3}=x^{2} \partial_{x}+2 x y \partial_{y}, \\
X_{1}=\partial_{x}+\partial_{y}, \quad X_{2}=x \partial_{x}+y \partial_{y}, \quad X_{3}=x^{2} \partial_{x}+y^{2} \partial_{y}, \\
X_{1}=\partial_{x}, \quad X_{2}=x \partial_{x}+y \partial_{y}, \quad X_{3}=\left(x^{2}-y^{2}\right) \partial_{x}+2 x y \partial_{y} .
\end{gathered}
$$

Therefore it is sufficient to restrict attention to ODEs that are invariant under one of the sets of generators (5.1)-(5.4). There are two ways in which an ODE may be invariant: either it can be rewritten in terms of the differential invariants, or else it is a singular variety where the orbit dimension drops and the Lie determinant vanishes (see Olver 1995). The latter case is of no interest to us, because the singular variety has Lie point symmetries other than those generated by one of (5.1)-(5.4). We refer to ODEs not of the singular variety as non-degenerate.

The fundamental differential invariants associated with (5.1) are

$$
I=y, \quad J=\frac{2 y^{\prime} y^{\prime \prime \prime}-3 y^{\prime \prime 2}}{2 y^{\prime 4}} .
$$

Every non-degenerate ODE that is invariant under (5.1) can be rewritten as an

Proc. R. Soc. Lond. A (1998) 
equation involving only $I, J$ and derivatives of $J$ with respect to $I$. If both $I$ and $J$ are invariant under a point transformation, then so are all derivatives of $J$ with respect to $I$, and thus the transformation is a point symmetry of every non-degenerate ODE whose continuous symmetries are generated by (5.1). It is straightforward to check, for each $B$ in (4.23), which of the point transformations satisfying (3.1) leave both $I$ and $J$ unchanged. This gives a list of four inequivalent symmetries of every non-degenerate ODE that is invariant under (5.1):

$$
(\hat{x}, \hat{y}) \in\{(x, y),(-x, y),(1 / x, y),(-1 / x, y)\} .
$$

The list is minimal; particular ODEs may have additional real discrete symmetries. For example, the ODE

$$
y^{\prime \prime \prime}=\frac{3 y^{\prime \prime 2}}{2 y^{\prime}}+y^{\prime 3} F\left(y^{2}\right)
$$

has a discrete symmetry

$$
(\hat{x}, \hat{y})=(x,-y)
$$

for any smooth function $F$. This symmetry is not equivalent to any of (5.6). In fact, $J$ is invariant under this symmetry, but $I$ is not - it is only a relative invariant (see Olver 1995).

In the same way, a minimal list of inequivalent real discrete symmetries can be constructed for the remaining actions of $S L(2)$ on the real plane. For brevity, we state only the fundamental differential invariants and the minimal list in each case.

The fundamental differential invariants corresponding to (5.2) are

$$
I=y y^{\prime \prime}-\frac{1}{2} y^{\prime 2}, \quad J=y^{2} y^{\prime \prime \prime},
$$

and the minimal list is

$$
(\hat{x}, \hat{y}) \in\left\{(x, y),(-x,-y),\left(1 / x,-y / x^{2}\right),\left(-1 / x, y / x^{2}\right)\right\} .
$$

The fundamental differential invariants corresponding to (5.3) are

$$
I=\frac{(y-x) y^{\prime \prime}-2 y^{\prime}\left(1+y^{\prime}\right)}{\left(y^{\prime}\right)^{3 / 2}}, \quad J=\frac{(y-x)^{2}\left(2 y^{\prime} y^{\prime \prime \prime}-3 y^{\prime 2}\right)}{2 y^{\prime 3}},
$$

and the minimal list is

$$
(\hat{x}, \hat{y}) \in\{(x, y),(-x,-y),(1 / x, 1 / y),(-1 / x,-1 / y)\} .
$$

The fundamental differential invariants corresponding to (5.4) are

$$
I=\frac{y y^{\prime \prime}+y^{\prime 2}+1}{y^{\prime 2}+1}, \quad J=\frac{y^{2}\left\{\left(y^{\prime 2}+1\right) y^{\prime \prime \prime}-3 y^{\prime} y^{\prime \prime 2}\right\}}{3\left(y^{\prime 2}+1\right)^{3}}
$$

and the minimal list is

$$
(\hat{x}, \hat{y}) \in\left\{(x, y),(-x,-y),\left(\frac{x}{x^{2}+y^{2}},-\frac{y}{x^{2}+y^{2}}\right),\left(-\frac{x}{x^{2}+y^{2}}, \frac{y}{x^{2}+y^{2}}\right)\right\} .
$$

These results show that every ODE whose continuous point symmetries are isomorphic to $S L(2)$ has four inequivalent real discrete symmetries.

Similar results can be proved for other Lie groups by the same method. It is not generally true that every symmetry of the Lie algebra of an ODE generates at least one discrete symmetry of the ODE. We have seen that discrete symmetries of (3.17) correspond to matrices $B$ of the special form (3.23), whereas any choice of non-singular $B$ preserves the structure constants in this example.

Proc. R. Soc. Lond. A (1998) 


\section{Final remarks}

The method introduced in this paper is easy to use, and is widely applicable. Nevertheless, it is only applicable to those ODEs that have non-trivial Lie groups of symmetries. For other ODEs, it seems that a reduction of the nonlinear determining equations to a differential Gröbner basis (using computer algebra) offers the best prospect of finding all discrete symmetries.

The new method can be extended to PDEs, as will be described in a later paper. Furthermore, it can be extended to other types of symmetries, such as contact symmetries (Hydon 1998). Contact symmetries can be used in similar ways to point symmetries, but they can be difficult to find and use (see Hydon 1996), and therefore they have been somewhat under-exploited. Nevertheless, discrete contact symmetries can be found systematically for ODEs of order three or greater. For example, Hydon (1998) demonstrates that the ODE (4.11) has eight inequivalent discrete contact symmetries. These are the four symmetries listed in (4.15) - point symmetries are special types of contact symmetries - and four more obtained by composing (4.15) with the Legendre transformation

$$
\Gamma_{2}:(x, y) \mapsto(\hat{x}, \hat{y})=\left(y^{\prime}, x y^{\prime}-y\right) .
$$

It remains to be seen how much further the method can be extended.

\section{References}

Allgower, E., Böhmer, K. \& Golubitsky, M. (eds) 1992 a Bifurcation and symmetry. Basel: Birkhäuser.

Allgower, E. L., Georg, K. \& Miranda, R. (eds) $1992 b$ Exploiting symmetry in applied and numerical analysis. Providence, RI: American Mathematical Society.

Bluman, G. W. \& Kumei, S. 1989 Symmetries and differential equations. New York: Springer.

Chadham, J. M., Golubitsky, M., Langford, W. \& Wetton, B. (eds) 1996 Pattern formation: symmetry methods and applications. Providence, RI: American Mathematical Society.

Clarkson, P. A. \& Olver, P. J. 1996 Symmetry and the Chazy equation. J. Differ. Equat. 124, 225-246.

Crawford, J. D., Golubitsky, M., Gomes, M. G. M., Knobloch, E. \& Stewart, I. N. 1991 Boundary conditions as symmetry constraints. In Singularity theory and its applications, Warwick 1989, part II (ed. M. Roberts \& I. Stewart), pp. 63-79. Berlin: Springer.

Gaeta, G. \& Rodríguez, M. A. 1996 Determining discrete symmetries of differential equations. Nuovo Cimento B 111, 879-891.

Golubitsky, M., Stewart, I. \& Schaeffer, D. G. 1988 Singularities and groups in bifurcation theory, vol. 2. New York: Springer.

Hydon, P. E. 1996 Self-invariant contact symmetries of second order ordinary differential equations. University of Surrey Technical Report 96/5/Ma.

Hydon, P. E. 1998 How to find discrete contact symmetries. University of Surrey Centre for Interdisciplinary Nonlinear Mathematics Technical Report 98/10.

Olver, P. J. 1986 Applications of Lie groups to differential equations. New York: Springer.

Olver, P. J. 1995 Equivalence, invariants and symmetry. Cambridge University Press.

Reid, G. J., Weih, D. T. \& Wittkopf, A. D. 1993 A point symmetry group of a differential equation which cannot be found using infinitesimal methods. In Modern group analysis: advanced analytical and computational methods in mathematical physics (ed. N. H. Ibragimov, M. Torrisi \& A. Valenti), pp. 311-316. Dordrecht: Kluwer.

Stephani, H. 1989 Differential equations: their solution using symmetries. Cambridge University Press.

Proc. R. Soc. Lond. A (1998) 